\title{
Preliminary results from simulations on the sub-galactic structure formation
}

\author{
Kyungwon Chun ${ }^{1}$ and Jihye Shin ${ }^{2}$ \\ ${ }^{1}$ School of Space Research, Kyung Hee University, Yongin, Gyeonggi, 446-701, Korea \\ email: kwchun@khu.ac.kr \\ ${ }^{2}$ Kavli Institute for Astronomy and Astrophysics, Peking University, 5 Yiheyuan Road, \\ Haidian District, Beijing 100871, China
}

\begin{abstract}
We aim to investigate the formation of sub-galactic structure in the Lambda cold dark matter (CDM) cosmology. To accomplish our research goal, we have added various baryonic physics on the existing cosmological hydrodynamic code, GADGET-2. We performed two test runs to check our new implementations. We show our preliminary results from these test runs.
\end{abstract}

Keywords. Galaxy: formation, large-scale structure of universe, globular clusters: general

\section{Introduction}

Our goal is to investigate the formation of sub-galactic structure in Lambda CDM cosmology. For this, we have modified the GADGET-2 (Springel 2005) code, a parallel $N$-body/SPH code, for the more realistic baryonic physics. We calculated radiative heating/cooling rates using CLOUDY90 package (Ferland et al. 1998). Global reionization is considered in the whole simulation volume at redshift $\mathrm{z}_{r e}=8.9$ (Haardt \& Madau 1996). We assume that the dense gas cloud $\left(\mathrm{n}_{H} \geqslant 0.014 \mathrm{~cm}^{-3}\right)$ (Sawala et al. 2010) is shielded from the universal UV radiation. Stars form when gas particles satisfy star formation criteria of Saitoh et al. (2008). The star particle is considered as a single stellar population with a spectrum of Salpeter mass function (Salpeter 1955). The number of stars that eventually ends up as type II supernovae is calculated using stellar evolutionary tracks of Hurley, Pols, \& Tout (2000). Energy, mass, and metals ejected by the supernovae are considered in a way to convey to its neighboring gas particles.

\section{Test runs}

We performed two test simulations that focus on an evolution of an isolated galaxy and study the cosmological structure formation. Table 1 gives parameters on the simulation. For the isolated galaxy, we were able to confirm that star formation rate (SFR) of isolated galaxy simulation well reproduces the Schmidt-Kennicutt law (Kennicutt 1998) (Fig. 1a). The cosmology is described with the cosmological parameters of $\Omega_{M}=0.276, \Omega_{\Lambda}=0.724$, $\Omega_{b}=0.045$, and $h=0.703$. Halos embedded in the more massive halos are identified using Rockstar halo finder (Behroozi, Wechsler, \& Wu 2013). Figure 1b shows that number density profile of subhalo around the main halo of $\sim 10^{10} M_{\odot}$ is described with a softened power law of $\alpha=3.3$. However, mass function is not consistent with the theoretical prediction of Sheth \& Tormen (1999), especially for the low halo mass (Fig. 1c). 
Table 1. Parameters of runs

\begin{tabular}{|c|c|c|c|c|c|c|c|}
\hline Model & $\mathbf{L}_{\mathrm{B} \text { ox }}{ }^{1}$ & $\left|\mathrm{~N}_{\mathrm{SPH}^{2}}{ }^{2}\right|$ & $\mathrm{m}_{\mathrm{SPH}}{ }^{3}$ & | Shielding ${ }^{4}$ & $\mathbf{n}_{\mathrm{th}^{5}}{ }^{5}$ & $\mathbf{T}_{\mathrm{th}}{ }^{6}$ & $\mathbf{C}_{*}{ }^{7}$ \\
\hline Isolated & $x$ & 98,304 & $\left|4.2 \times 10^{4} M_{\odot}\right|$ & $x$ & $0.1 \mathrm{~cm}^{-3}$ & $15000 \mathrm{~K}$ & $\mid 0.033$ \\
\hline Run 1 & $8 \mathrm{Mpc} / \mathrm{h}$ & $256^{3}$ & $\left|3.8 \times 10^{5} M_{\odot}\right|$ & $\bigcirc$ & $0.1 \mathrm{~cm}^{-3}$ & $15000 \mathrm{~K}$ & 0.033 \\
\hline Run 2 & $8 \mathrm{Mpc} /$ & $256^{3}$ & $3.8 \times 10^{5} M_{\odot}$ & 0 & $100 \mathrm{~cm}^{-3}$ & $5000 K$ & | 0.033 \\
\hline
\end{tabular}

${ }^{1}$ A side length of a cube box ${ }^{2}$ Number of gas particles ${ }^{3}$ Mass of gas particles

${ }^{4}$ UV Shielding ${ }^{5}$ Threshold number density of star formation

${ }^{6}$ Threshold Temperature of star formation ${ }^{7}$ Star formation efficiency
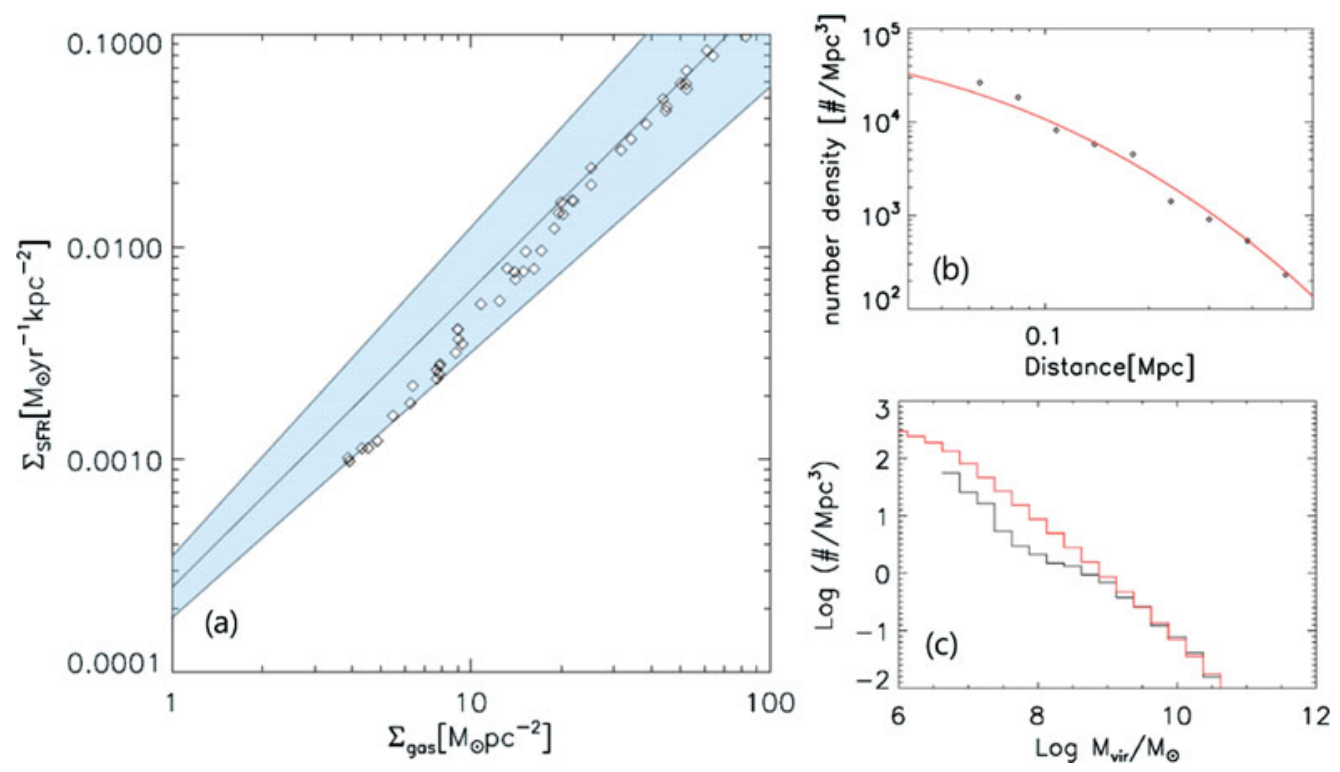

Figure 1. Results of runs. (a) The isolated galaxy simulation represents Schmidt-Kennicutt law. (b) Spatial density profile can be fitted with softened power law ( $\alpha=3.3)$. (c) Dark matter mass function with the theoretical mass function(red line) and the simulation result(black line).

\section{Future work}

We will use more realistic power spectrum and halo-finding algorithm. After confirming our modified GADGET-2 code is ready for the scientific studies, we will perform a multilevel simulation that resolves sub-galactic structure around the Milky Way-like main halo.

\section{References}

Behroozi, P. S., Wechsler, R. H. \& Wu, H. Y. 2013a, ApJ, 762, 109

Ferland, G. J., Korista, K. T., Verner, D. A., Ferguson, J. W., Kingdon, J. B., \& Verner, E. M. 1998, PASP, 110, 761

Haardt, F. \& Madau, P. 1996, ApJ, 461, 20

Hurley, J. R., Pols, O. R., \& Tout, C. A. 2000, MNRAS, 315, 543

Kennicutt, R. C.,Jr. 1998, ApJ, 498,541

Saitoh, T. R., Daisaka, H., Kokubo, E., Makino, J., Okamoto, T., Tomisaka, K., Wada, K., \& Yoshida, N. 2008, PASJ, 60, 667

Salpeter, E. E. 1955, ApJ, 121, 161

Sawala, T., Scannapieco, C., Maio, U., \& White, S. 2010, MNRAS, 402, 1599

Springel, V. 2005, MNRAS, 364, 1105 